\title{
Avaliação da taxa de filtração glomerular em idosos
}

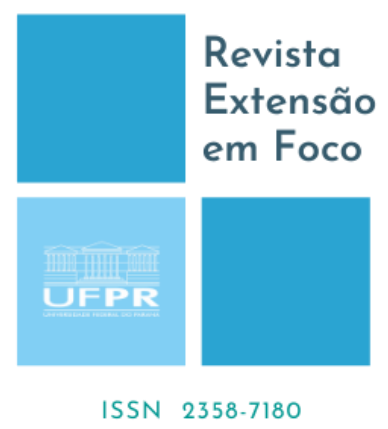

\author{
Evaluation of the estimated glomerular filtration \\ rate in elderlyl
}

\author{
Paloma de Morais ${ }^{1}$, Stella Bortoli ${ }^{2}$, Margarete Aparecida Salina Maciel $^{3}$, Danielle \\ Cristyane Kalva Borato ${ }^{4}$
}

\begin{abstract}
RESUMO
A determinação mais confiável para avaliação da função renal é a taxa de filtração glomerular (TFG). Devido às limitações encontradas com a determinação da TFG pelo clearance de creatinina ou pela creatinina sérica, foram criadas fórmulas para estimar com maior precisão a função renal. O projeto de extensão "Contribuição laboratorial à saúde em uma instituição de longa permanência de idosos de Ponta Grossa - Paraná", vinculado à Universidade Estadual de Ponta Grossa realizou uma ação com objetivo de verificar a função renal pela taxa de filtração glomerular estimada (TFGe), utilizando a equação BISCreatinina. Foram avaliados 94 idosos com idade média de $76 \pm 8$ anos, sendo 56 (60\%) do gênero feminino. Os idosos foram alocados em três grupos, conforme a faixa etária: 60 - 69 anos $(\mathrm{n}=21 ; 22 \%)$, $70-79$ anos $(\mathrm{n}=43 ; 46 \%)$ e $\geq 80$ anos $(\mathrm{n}=30 ; 32 \%)$. Os resultados da TFGe foram avaliados pelos valores referenciais, segundo o Kidney Disease Outcomes Quality Initiative (KDIGO) visando o planejamento de intervenções para retardar a progressão da doença renal e a prescrição correta dos medicamentos utilizados pelos idosos. A maioria dos idosos apresentou redução discreta da TFGe. Verificou-se 5\% dos idosos com redução severa da TFGe nos grupos com 60 - 69 anos e $70-79$ anos. O grupo $\geq 80$ anos apresentou maior percentual de indivíduos com redução discreta-moderada $(25 \%)$ e moderada-severa (18\%), não sendo observada redução severa da TFGe. Assim, a atividade extensionista desenvolvida auxiliou no diagnóstico clínico dos idosos e no aperfeiçoamento acadêmico dos alunos participantes do projeto.
\end{abstract}

Palavras-chave: Idosos. Envelhecimento. Função Renal. Taxa de Filtração Glomerular.

\section{ABSTRACT}

The most reliable determination for renal function assessment is glomerular filtration rate (GFR). Due to the limitations found with the determination of GFR by creatinine clearance or serum creatinine, formulas were created to more accurately estimate the renal function. The extension project "Laboratory contribution to health in a long-term care facility for elderly in Ponta Grossa - Paraná", linked to the Ponta Grossa State University, carried out an action to verify renal function by estimated glomerular filtration rate (eGFR) using the BIS-creatinine equation. Ninety-four elderly individuals with a mean age of $76 \pm 8$ years were evaluated, $56(60 \%)$ females. The elderlies were allocated into three groups, according to age: 60 - 69 years $(n=21 ; 22 \%), 70-79$ years $(n=43 ; 46 \%)$ and $\geq 80$ years $(n=30 ; 32 \%)$. The eGFR results were evaluated by reference values, according to the Kidney Disease Outcomes Quality Initiative (KDIGO) aiming the planning of interventions to slow the progression of kidney disease and the correct prescription of medications used by the elderlies. Most of the elderly showed a slight reduction in

\footnotetext{
${ }^{1}$ Graduada. Universidade Estadual de Ponta Grossa (UEPG), Ponta Grossa, Paraná, Brasil. E-mail: moraispah@ hotmail.com. Orcid: https://orcid.org/0000-0001-5548-1583

${ }^{2}$ Doutora. Universidade Estadual de Ponta Grossa (UEPG), Ponta Grossa, Paraná, Brasil. E-mail: stellabortoli@gmail.com. Orcid: https://orcid.org/0000-0003-0636-4692

${ }^{3}$ Doutora. Universidade Estadual de Ponta Grossa (UEPG), Ponta Grossa, Paraná, Brasil. E-mail: mmaciel2020@ gmail.com. Orcid: https://orcid.org/0000-0001-5688-6195

${ }^{4}$ Doutora. Universidade Estadual de Ponta Grossa (UEPG), Ponta Grossa, Paraná, Brasil. E-mail: dckalva@ hotmail.com. Orcid: https://orcid.org/0000-0002-9987-6250
} 
eGFR. It was verified 5\% of the elderly with severe eGFR reduction in the groups with $60-69$ years and $70-79$ years. The $\geq 80$-year group showed a higher percentage of individuals with mild-moderate $(25 \%)$ and moderate-severe $(18 \%)$ reduction, with no severe reduction in eGFR. So that, the extension activity developed helped in the clinical diagnosis of the elderly and in the academic improvement of the students participating in the extension project. Prepare the abstract a single paragraph, minimum 150 and maximum 250 words, including objective, methodology, results and conclusions. The objective should be short, defining the problem studied, highlighting the knowledge gaps that will be addressed in the article. Data sources, study population, sampling, selection criteria, analytical procedures, among others, must be described in a comprehensive and complete. The Results section should be limited to describing the results without including interpretations/comparisons. The authors' conclusion on the results and their main implications.

Keywords: Elderly. Aging. Kidney function. Glomerular Filtration Rate.

\section{INTRODUÇÃO}

O envelhecimento é considerado um fator de risco para doença renal crônica (ABDULKADER et al., 2017). Portanto, a monitoração precisa da função renal em idosos é de considerável interesse clínico, a fim de detectar indivíduos que estão em risco de desenvolver a doença renal crônica (FLISER, 2008). Além disso, a avaliação da função renal é um pré-requisito para o manejo farmacológico correto em idosos (DESKUR-SMIELECKA et al., 2017).

A determinação mais confiável para avaliação da função renal é a taxa de filtração glomerular (TFG) (CLARO; STINGHEN; PECOITS-FILHO, 2011). No entanto, na prática clínica o nível sérico de creatinina é o marcador de função renal mais utilizado e o clearance de creatinina urinário de 24 horas é considerado como um marcador substituto da TFG (PORRINI et al., 2019). Porém, a depuração de creatinina pode superestimar a verdadeira TFG uma vez que a creatinina é excretada não apenas via filtração glomerular, mas também via secreção no túbulo proximal (FERGUSON; WAIKAR, 2012). Assim, a secreção tubular de creatinina é aumentada proporcionalmente em relação à sua filtração glomerular, à medida que a função renal diminui resulta em uma superestimação significativa da TFG (FERGUSON; WAIKAR, 2012). Além da imprecisão do método de depuração da creatinina para medir a TFG, o principal problema para os idosos é a exigência da coleta de urina em 24 horas, muitas vezes complexa (BASTOS; KIRSZTAJN, 2011).

Além disso, com o envelhecimento ocorre um declínio na TFG, devido à perda de massa muscular relacionada à idade. No entanto, não há aumento concomitante da 
creatinina sanguínea (MANGONI; JACKSON, 2004). Portanto, a creatinina sérica não é um indicador confiável da taxa de filtração glomerular em idosos, particularmente naqueles que estão doentes e/ou desnutridos (FLISER, 2008).

Devido às limitações encontradas com a determinação da TFG pelo clearance de creatinina ou pela creatinina sérica, foram criadas fórmulas utilizando a concentração sérica da creatinina, assim como outros dados clínicos e laboratoriais para estimar com maior precisão a TFG (BASTOS; KIRSZTAJN, 2011). A Kidney Disease Outcomes Quality Initiative (KDIGO) recomenda a utilização da equação proposta pela Chronic Kidney Disease Epidemiology ([Epidemiologia da Doença Renal Crônica] [CKD-EPI]) para estimar TFG (MILLER; JONES, 2018). No entanto, essa fórmula não foi projetada especificamente para pessoas idosas, enquanto as equações desenvolvidas pelo Berlin Initiative Study (BIS) demonstram boa acurácia em indivíduos idosos, como a equação BIS-creatinina, "BIS-1" (SCHAEFFNER et al., 2012).

Segundo Fliser (2008) as estimativas mais confiáveis da TFG devem ser empregadas, sempre que possível, na população idosa. Nesse sentido, o projeto de extensão "Contribuição laboratorial à saúde em uma instituição de longa permanência de idosos de Ponta Grossa - Paraná" vinculado a Universidade Estadual de Ponta Grossa (UEPG), realizou uma ação extensionista com objetivo de verificar a função renal, pela taxa de filtração glomerular estimada (TFGe), projetada especificamente para pessoas idosas, por meio da equação BIS-Creatinina, em indivíduos residentes em uma Instituição de Longa Permanência de Idosos (ILPI).

\section{MÉTODOS}

O projeto é realizado por alunos e professores do curso de Farmácia da UEPG, visando a indissociabilidade entre ensino, pesquisa e extensão. Atualmente são atendidos pelo projeto cerca de 110 indivíduos, de ambos os gêneros, masculino e feminino, na maioria com idade acima de 50 anos que residem na ILPI, instituição filantrópica conhecida como Asilo São Vicente de Paulo, no município de Ponta Grossa, Paraná.

A ação extensionista foi realizada na própria ILPI, com frequência semanal, visando a integração entre a universidade com a comunidade de idosos residentes na 
instituição e o atendimento laboratorial dos participantes do projeto. Enquanto que, as análises laboratoriais foram realizadas pelos alunos do $5^{\circ}$ ano do curso de Farmácia, com supervisão dos professores responsáveis, no Laboratório Universitário de Análises Clínicas (LUAC), da UEPG.

Neste estudo foram incluídos indivíduos participantes do projeto de extensão com idade igual ou superior a 60 anos, que realizaram a dosagem de creatinina no ano de 2018. As informações pessoais dos participantes da pesquisa, como idade e gênero, foram obtidas do prontuário médico fornecido pela instituição. Os idosos foram submetidos a coleta de sangue por punção venosa, após jejum de 8 a 12 horas. A seguir, o sangue foi colocado em tubo sem anticoagulante e processado obtendo-se o soro, que foi utilizado para a avaliação da creatinina sérica. As concentrações séricas de creatinina foram obtidas pelo método cinético em aparelho automatizado CT 300i da WIENER (Wiener Lab. Group®), no setor de bioquímica do LUAC.

Como os valores de creatinina sérica foram obtidos por método enzimático não rastreável ao ID-MS (Isothope Dillution Mass Spectometry), considerado padrão ouro para dosagem de creatinina, os resultados obtidos foram padronizados de acordo com o método referência, pela seguinte equação: Creatinina_ID-MS (mg/dL) = (Creatinina_convencional - 0,067)/1,065. A TFGe foi avaliada de acordo com Berlin Initiative Study (BIS), obtida pela seguinte fórmula: BIS-Creatinina "BIS-1" = $3736 *$ Creatinina ${ }^{-0,87 *}$ Idade $^{-0,95}$, sendo que para os participantes do gênero feminino a TFGe foi multiplicada por 0,82 .

Como ocorre uma diminuição fisiológica da TFG com a idade, os idosos foram alocados em três grupos, de acordo com a faixa etária, para avaliação da TFGe utilizando a equação BIS-Creatinina: i) idosos com 60 - 69 anos, ii) idosos com 70 - 79 anos e iii) idosos com idade superior a 80 anos.

Os resultados da TFGe foram avaliados pelos valores referenciais e estadiamento, segundo o KDIGO, para adultos: normal > $90 \mathrm{~mL} / \mathrm{min}$ x 1,73m² (estádio G1), redução discreta $89-60 \mathrm{ml} / \mathrm{min} \times 1,73 \mathrm{~m}^{2}$ (estádio G2), redução discreta-moderada 59 - 45 $\mathrm{ml} / \mathrm{min} \times 1,73 \mathrm{~m}^{2}$ (estádio G3a), redução moderada-severa $44-30 \mathrm{~mL} / \mathrm{min} \times 1,73 \mathrm{~m}^{2}$ (estádio G3b), redução severa 29 - $15 \mathrm{~mL} / \mathrm{min}$ x 1,73m² (estádio G4), falência renal < $15 \mathrm{~mL} / \mathrm{min} \times 1,73 \mathrm{~m}^{2}$ (estádio G5) (WEBSTER et al., 2017) 
Este estudo foi realizado em conformidade com a Declaração de Helsinki e aprovado pelo Comitê de Ética em Pesquisa da Universidade Estadual de Ponta Grossa, parecer $n^{\circ} 2.745 .328 / 18$.

\section{RESULTADOS E DISCUSSÃO}

Foram avaliados na atividade de extensão 94 idosos com idade média de $76 \pm 8$ anos, sendo $56(60 \%)$ dos indivíduos do gênero feminino e 38 (40\%) do gênero masculino. O grupo com faixa etária entre 60 e 69 anos constituiu-se de 21 (22\%) idosos; enquanto que, o grupo entre 70 e 79 anos apresentou 43 (46\%) idosos e o grupo com idade superior a 80 anos foi composto por 30 (32\%) idosos.

A doença renal crônica é considerada um problema de saúde pública, devido sua prevalência que vem crescendo nos últimos anos, atingindo todas as faixas etárias (MARINHO et al., 2017). Informações da Pesquisa Nacional de Saúde e Nutrição (NHANES 2007-2012) demonstraram prevalência de doença renal crônica de 5,7\%, $8,9 \%$ e $33,2 \%$ nas faixas etárias de 20 a 39,40 a 59 e $\geq 60$ anos, respectivamente (ABDULKADER et al., 2017). Deve-se salientar que na maior parte dos casos, o diagnóstico não é realizado nos estágios iniciais da doença, visto que a doença renal é silenciosa (EKNOYAN et al., 2004). Sendo assim, nos indivíduos com maior possibilidade de avanço da doença, como nos idosos, é fundamental reconhecer as alterações da função renal em sua fase prematura (SESSO et al., 2014).

Os resultados da função renal pela TFGe obtidos pela fórmula BIS-Creatinina, segundo os valores referenciais e o estadiamento pelo KDIGO, nos idosos com 60 a 69 anos, 70 a 79 anos e com mais de 80 anos estão demonstrados nas Figuras 1, 2 e 3, respectivamente.

Observou-se que o grupo de idosos com idade entre 60 e 69 anos apresentou o maior percentual $(24 \%)$ de indivíduos com a TFGe normal, em comparação com os outros grupos. No entanto, a maioria dos idosos avaliados no estudo apresentou redução discreta da TFGe, independente da faixa etária avaliada. Além disso, verificou-se $5 \%$ de indivíduos com redução severa da TFGe, tanto para o grupo de idosos com idade entre 60 e 69 anos como para o grupo de idosos com idade de 70 a 79 anos. Porém, não foi observado redução severa da função renal em idosos com idade superior a 80 anos, 
ressaltando que esse grupo apresentou o maior percentual de indivíduos com redução discreta-moderada (25\%) e moderada-severa (18\%) da função renal.

Figura 1 - Percentual de idosos com 60 a 69 anos atendidos no projeto de extensão de acordo com a função renal

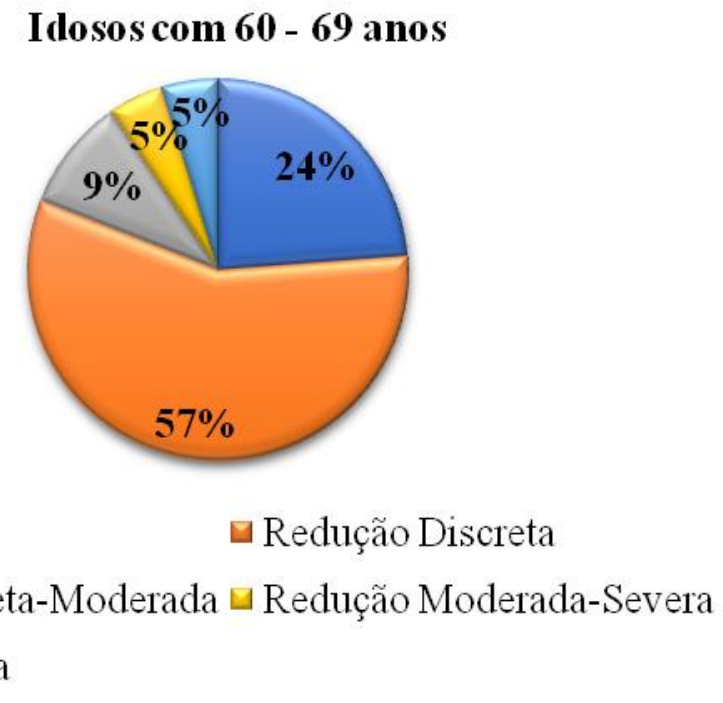

Fonte: Autoria própria (2018).

Figura 2 - Percentual de idosos com 70 a 79 anos atendidos no projeto de extensão de acordo com a função renal

\section{Idosos com 70 a 79 anos}

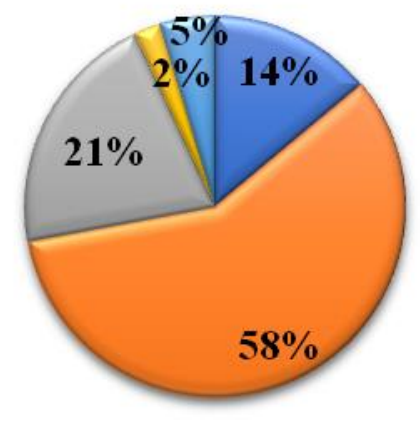

Normal

$\square$ Redução Discreta

$\square$ Redução Discreta-Moderada $\square$ Redução Moderada-Severa $\square$ Redução Severa

Fonte: Autoria própria (2018). 
Figura 3 - Percentual de idosos com mais de 80 anos atendidos no projeto de extensão de acordo com a função renal

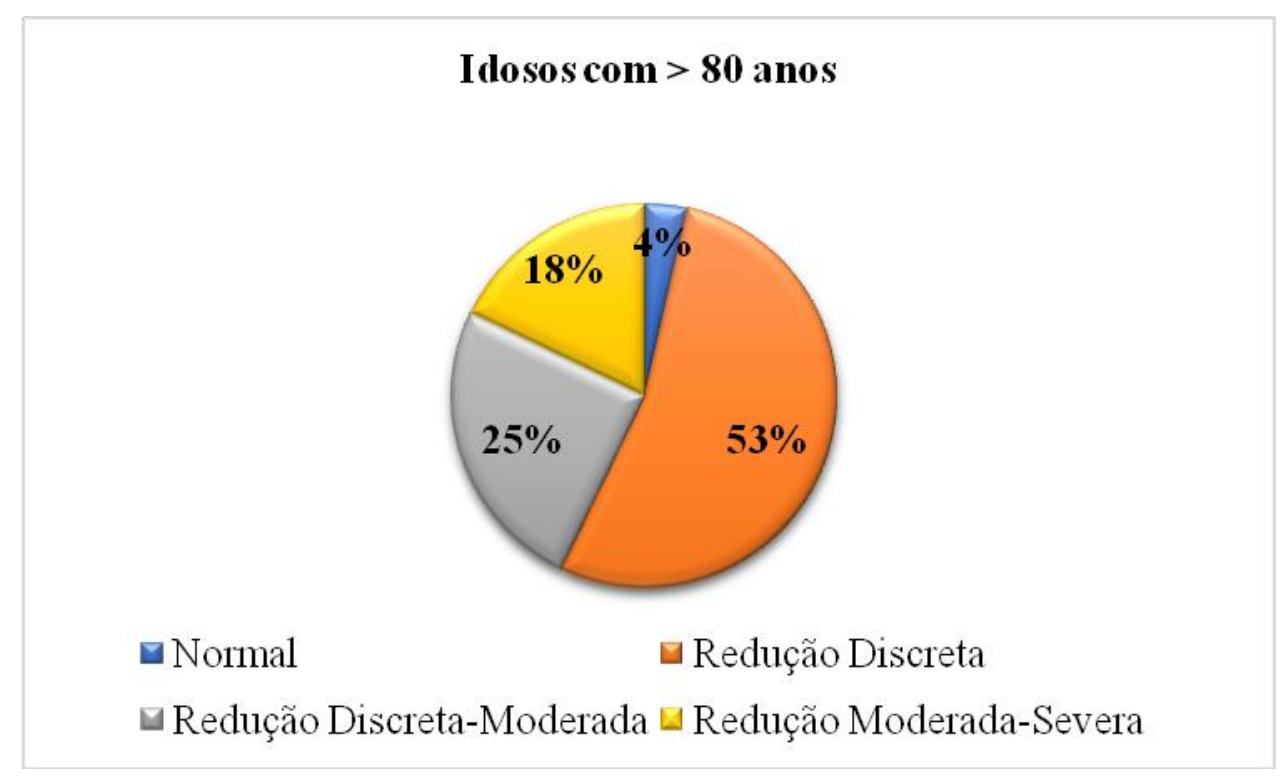

Fonte: Autoria própria (2018).

Estudos avaliando a TFGe com fórmulas utilizando creatinina sérica na população idosa são escassos. Segundo Schaeffner et al. (2012), o objetivo do Berlin Initiative Study (BIS) foi avaliar a função renal em uma coorte de base populacional idosa comparando as equações existentes com uma medida padrão-ouro. Isso foi clinicamente relevante, pois faltava uma equação validada para estimar a TFG em adultos mais velhos, especialmente em casos de função renal normal ou apenas moderadamente reduzida, diminuindo a classificação incorreta de indivíduos com TFG maior ou menor de $60 \mathrm{~mL} / \mathrm{min} / 1,73 \mathrm{~m}^{2}$ (SCHAEFFNER et al., 2012).

Um estudo retrospectivo desenvolvido por Koppe et al. (2013) comparou a performance da equação BIS-Creatinina com as equações CKD-EPI e MDRD (Modification of Diet in Renal Disease) $e$ demonstrou que a BIS-Creatinina é mais exata e precisa na avaliação da TFG, especialmente com TFG $>30 \mathrm{~mL} / \mathrm{min} / 1,73 \mathrm{~m}^{2}$. Porém, a equação CKD-EPI é mais precisa para TFG $<30 \mathrm{~mL} / \mathrm{min} / 1,73 \mathrm{~m}^{2}$. Contudo, para avaliação dos idosos com função renal normal é necessário estudos adicionais para a equação BIS-Creatinina, visto que este incluiu apenas indivíduos com suspeita ou disfunção renal estabelecida, no que se referem os autores (KOPPE et al., 2013).

Outro estudo transversal retrospectivo, que envolveu 600 indivíduos com mais de 85 anos, a fórmula BIS-Creatinina estimou valores mais baixos de TFG que as equações 
CKD-EPI e MDRD e apresentou tendência a classificar os pacientes em um estágio mais avançado da doença renal crônica, especialmente para TFGe maior que 29 $\mathrm{mL} / \mathrm{min} / 1,73 \mathrm{~m}^{2}$ (BUSTOS-GUADAÑO et al., 2017).

Uma recente revisão sistemática envolvendo 1.295 estudos demonstrou que, para a estimativa da TFG em idosos, a fórmula do BIS-Creatinina é mais precisa que CKDEPI (OSCANOA et al., 2018). Portanto, estes estudos demonstram a importância da avaliação da função renal em idosos por meio da fórmula específica para essa população, a BIS-Creatinina.

Além disso, deve ser levando em consideração que no processo normal de senescência morfológica ocorre um declínio do fluxo renal sanguíneo, associado a alterações estruturais e funcionais nos rins (KARAM; TUAZON, 2013). Indivíduos com mais de 40 anos perdem, em geral, perto de $1 \mathrm{~mL} / \mathrm{min}$ da TFG por ano, devido a perdas de néfrons, como parte do processo de envelhecimento. Por conseguinte, uma TFGe menor do que $60 \mathrm{~mL} / \mathrm{min} / 1,73 \mathrm{~m}^{2}$ nem sempre é indicativo de doença renal crônica para indivíduos com mais de 70 anos. Assim como, em um idoso de 80 anos pode-se esperar uma TFGe de $45-50 \mathrm{~mL} / \mathrm{min} / 1,73 \mathrm{~m}^{2}$ (SOCIEDADE BRASILEIRA DE NEFROLOGIA, 2015).

Portanto, mesmo que ocorra declínio da função renal com o aumento da idade atribuído ao envelhecimento fisiológico, nem todos os idosos desenvolvem doença renal crônica (DENIC; GLASSOCK; RULE, 2016). Além disso, existem diversas condições que podem danificar ou intensificar o comprometimento da função renal, tais como diabetes mellitus e hipertensão arterial sistêmica (BASTOS; KIRSZTAJN, 2011). Ao mesmo tempo, a predisposição genética e exposição a condições ambientais, tais como metais pesados (chumbo, cádmio, arsênico, mercúrio, urânio), produtos químicos ambientais e agrícolas, resíduos industriais, medicamentos anti-inflamatórios não esteroides, medicamentos falsificados, fitoterápicos tradicionais, infecções, consumo excessivo de álcool, bebidas açucaradas e excesso de sal podem aumentar a probabilidade do desenvolvimento de doença renal crônica (OBRADOR et al., 2017).

Desta forma, os resultados da TFGe foram avaliados de forma criteriosa pelos professores e estudantes participantes do projeto de extensão, para avaliar a possibilidade de incluir a equação BIS-Creatinina na prática clínica, visando o 
planejamento de intervenções que possam retardar a progressão da doença renal e a prescrição apropriada da dose dos medicamentos.

No presente trabalho avaliou-se a função renal, pela TFGe específica para idosos, buscando uma adequada investigação laboratorial e um acompanhamento farmacológico e nefrológico adequado dos idosos, contribuindo para uma melhor qualidade de vida destes indivíduos. Desta forma, as atividades extensionistas desenvolvidas pelo projeto têm auxiliado no diagnóstico e acompanhamento clínico dos idosos residentes na ILPI e no aperfeiçoamento acadêmico dos alunos envolvidos com o projeto.

\section{AGRADECIMENTOS E APOIO}

Os autores são gratos a Pró-Reitoria de Extensão e Assuntos Culturais da UEPG/Fundação Araucária, ao Asilo São Vicente de Paulo (Instituição de Longa Permanência de Idosos - ILPI) e ao LUAC - Laboratório Universitário de Análises Clínicas por aprovarem a realização desta pesquisa.

\section{REFERÊNCIAS}

ABDULKADER, R. C. R. M. et al. Aging and decreased glomerular filtration rate: An elderly population-based study. PLoS One, v. 12, n. 12, p. 1-12, 2017.

BASTOS, M. G.; KIRSZTAJN, G. M. Chronic kidney disease: importance of early diagnosis, immediate referral and structured interdisciplinary approach to improve outcomes in patients not yet on dialysis. J. Bras. Nefrol., v. 33, n. 1, p. 93-108, 2011.

BUSTOS-GUADAÑO, F. et al. Glomerular filtration rate estimation in people older than 85: Comparison between CKD-EPI, MDRD-IDMS and BIS1 equations. Nefrologia, v. 37, n. 2, p. 172-180, 2017.

CLARO, L.; STINGHEN, A. E.; PECOITS-FILHO, R. Biomarcadores de Inflamação na Doença Renal Crônica. Biomarcadores na Nefrologia, p. 81-92, 2011.

DENIC, A.; GLASSOCK, R. J.; RULE, A. D. Structural and Functional Changes With the Aging Kidney. Adv. Chronic Kidney Dis., v. 23, n. 1, p. 19-28, 2016.

DESKUR-SMIELECKA, E. et al. Assessment of renal function in geriatric palliative care patients - comparison of creatinine-based estimation equations. Clin. 
Interven. Aging, v. 12, p. 977-983, 2017.

EKNOYAN, G. et al. The burden of kidney disease: improving global outcomes. Kidney Int., v. 66, n. 4, p. 1310-1314, out. 2004.

FERGUSON, M. A.; WAIKAR, S. S. Established and emerging markers of kidney function. Clin. Chem., v. 58, n. 4, p. 680-689, abr. 2012.

FLISER, D. Assessment of renal function in elderly patients. Curr. Opin. Nephrol. Hypertens., v. 17, n. 6, p. 604-608, nov. 2008.

KARAM, Z.; TUAZON, J. Anatomic and physiologic changes of the aging kidney. Clin. Geriatr. Med., v. 29, n. 3, p. 555-564, ago. 2013.

KOPPE, L. et al. Performance of creatinine-based equations compared in older patients. J. Nephrol., v. 26, n. 4, p. 716-723, 2013.

MANGONI, A. A.; JACKSON, S. H. D. Age-related changes in pharmacokinetics and pharmacodynamics: basic principles and practical applications. Br. J. Clin. Pharmacol, v. 57, n. 1, p. 6-14, jan. 2004.

MARINHO, A. W. G. B. et al. Prevalência de doença renal crônica em adultos no Brasil: revisão sistemática da literatura. Cad. Saúde Colet., v. 25, n. 3, p. 379-388, 2017.

MILLER, W. G.; JONES, G. R. D. Estimated Glomerular Filtration Rate; Laboratory Implementation and Current Global Status. Adv. Chronic Kidney Dis., v. 25 , n. 1, p. 7-13, jan. 2018.

OBRADOR, G. T. et al. Genetic and environmental risk factors for chronic kidney disease. Kidney Int. Suppl., v. 7, n. 2, p. 88-106, 2017.

OSCANOA, T. J. et al. Estimation of the glomerular filtration rate in older individuals with serum creatinine-based equations: A systematic comparison between CKD-EPI and BIS1. Arch. Gerontol. Geriatr., v. 75, n. June 2017, p. 139-145, 2018.

PORRINI, E. et al. Estimated GFR: time for a critical appraisal. Nat. Rev. Nephrol., v. 15, n. 3, p. 177-190, mar. 2019.

SCHAEFFNER, E. S. et al. Two novel equations to estimate kidney function in persons aged 70 years or older. Ann. Intern. Med., v. 157, n. 7, p. 471-481, out. 2012. 
SESSO, R. C. INTR. et al. Report of the Brazilian Chronic Dialysis Census 2012. Jornal brasileiro de nefrologia : orgão oficial de Sociedades Brasileira e LatinoAmericana de Nefrologia, v. 36, n. 1, p. 48-53, 2014.

SOCIEDADE BRASILEIRA DE NEFROLOGIA. Passo a passo para a implantação da estimativa da taxa de filtração glomerular (eTFG). Sociedade Brasileira de Patologia Clínica, p. 1-11, 2015.

WEBSTER, A. C. et al. Chronic Kidney Disease. Lancet, v. 389, n. 10075, p. 1238-1252, 2017.

Recebido em: 30 de outubro de 2019. Aceito em: 06 de maio de 2020. 